\title{
Determinants of quality of life in patients with breast cancer undergoing external beam radiotherapy
}

Justyna Chalubinska-Fendler ${ }^{1}$, Agnieszka Butwicka² ${ }^{2}$ Patrycja Kaźmierczak ${ }^{1}$, Anna Jałocha-Kaczka ${ }^{3}$, Wojciech Fendler ${ }^{4}$, Jacek Fijuth ${ }^{1}$

'Department of Radiotherapy, Medical University of Lodz, Lodz, Poland

${ }^{2}$ Department of Adolescent Psychiatry, Medical University of Warsaw, Warsaw, Poland ${ }^{3}$ Department of Otolaryngology and Laryngeal Oncology, Medical University of Lodz, Lodz, Poland

${ }^{4}$ Department of Pediatrics, Oncology, Hematology and Diabetology, Medical University of Lodz, Lodz, Poland

Submitted: 18 March 2014

Accepted: 21 March 2014

Arch Med Sci 2015; 11, 6: 1361-1362

DOI: $10.5114 /$ aoms.2015.56367

Copyright @ 2015 Termedia \& Banach

Quality of life (QoL) of patients with breast cancer is not dependent only on the presence of the disease, as there is such a multitude of social and clinical factors [1, 2]. However, data on the determinants of QoL in Polish breast cancer patients during the course of radiotherapy are scarce and based on custom-made questionnaires [3], rather than the EORTC QLQ-C30 or QLQ-BR23, which are the tools of choice for such patients $[4,5]$. We evaluated breast cancer patients undergoing radiotherapy for treatment-related determinants of QoL and compared them with the intensity of depressive symptoms.

A total of 98 women with breast cancer during the standard course of external beam radiotherapy agreed to complete the validated Polish versions 3.0 of EORTC QLQ-C30, QLQ-BR23 (all global, functional and symptom scales) and - at the same time point - the Beck Depression Inventory (BDI) to evaluate the intensity of depressive symptoms. A control group of 127 healthy women referred to the mammography department for screening purposes was evaluated using the BDI.

Median age of the study group was 54 (25-75\%: 46.7-60.5) years. Breast conserving therapy (BCT) had been performed in $50 \%$ of patients. In the breast cancer group, $65 \%$ of subjects had undergone some form of chemotherapy and $51 \%$ were undergoing hormone therapy. Cronbach's $\alpha$ values for QLQ-C30, QLQ-BR23 and BDI were 0.91, 0.81 and 0.83 respectively, confirming adequate test performance.

Median total radiation dose given to the patients was $34 \mathrm{~Gy}$ (25-75\%: 18-42 Gy), and only affected intensity of nausea/vomiting and constipation subscales $(R=0.25, p=0.04 ; R=0.27, p=0.03$ respectively) of the QLQ-C30 functional scales. No statistically significant correlations were found between the total dose and QLQ-BR23 scales, but the early reactions (RTOG staged $\geq 2$ [6]) significantly worsened arm symptom subscale score assessment in QLQ-BR23.

Hormonal therapy was not significantly correlated with any of those three questionnaires' subscores. Previously received chemotherapy impacted neither QLQ-C30 nor BDI results, but it was associated with body image (BRBI) and systemic therapy side effect scores (BRST) $(p=0.02$ and $p=0.005$ ). Type of surgical intervention before radiotherapy impacted the field of global health status, resulting in better overall QoL in

\author{
Corresponding author: \\ Justyna Chalubinska-Fendler \\ MD \\ Department of Radiotherapy \\ Medical University of Lodz \\ 4 Paderewskiego St \\ 93-509 Lodz, Poland \\ Phone: +48 426895405 \\ Fax: +48 426895550 \\ E-mail: justyna.chalubinska- \\ fendler@e.umed.lodz.pl
}


patients who had undergone BCT (58\% (25-75\%: $50-67)$ vs. $50 \%$ (25-75\%: 42-58); $p=0.0405)$. BCT was also associated with lower reported intensity of breast symptoms $(p=0.0338)$ and better perception of future perspectives (BRFU; $p=0.0328$ ). The level of depressive symptoms measured in controls was significantly lower than in cancer patients (median 5 (25-75\%: 1-10) vs. 12 (25-75\%: 6-17) points; $p<0.0001)$. BDI scores were correlated negatively and significantly with all functional scales of QLQ-C30 (Spearman's correlation coefficients ranging from -0.36 to -0.46 , all $p<0.05)$ and QLQ-BR23 subscales: BRBI and $\operatorname{BRFU}(R=-0.47$ and $-0.51 ; p<0.05)$.

In conclusion, radiotherapy itself has a minor influence on QoL of breast cancer patients, although organ-specific complications may significantly impair physical functioning. Other forms of oncological treatment, such as the type of surgical procedure performed, have a much more profound impact on all fields of QoL. Intensity of depressive symptoms is an important determinant of QoL in cancer patients which mandates routine psychological evaluation.

\section{Acknowledgments}

This publication was financed from National Science Centre grant "Preludium” No. 2012/05/N/ NZ5/02621.

\section{Conflict of interest}

The authors declare no conflict of interest.

\section{References}

1. Bowen DJ, Alfano CM, McGregor BA, et al. Possible socioeconomic and ethnic disparities in quality of life in a cohort of breast cancer survivors. Breast Cancer Res Treatment 2007; 106: 85-95.

2. Lemieux J, Maunsell E, Provencher L. Chemotherapy-induced alopecia and effects on quality of life among women with breast cancer: a literature review. Psychooncology 2008; 17: 317-28.

3. Pacian A, Kulik TB, Pacian J, et al. Psychosocial aspect of quality of life of Polish women with breast cancer. Ann Agric Environ Med 2012; 19: 509-12.

4. Aaronson NK, Ahmedzai S, Bergman B, et al. The European Organization for Research and Treatment of Cancer QLQ-C30: a quality-of-life instrument for use in international clinical trials in oncology. J Natl Cancer Instit 1993; 85: 365-76.

5. Sprangers MA, Groenvold M, Arraras JI, et al. The European Organization for Research and Treatment of Cancer breast cancer-specific quality-of-life questionnaire module: first results from a three-country field study. J Clin Oncol 1996; 14: 2756-68.

6. Cox JD, Stetz J, Pajak TF. Toxicity criteria of the Radiation Therapy Oncology Group (RTOG) and the European Organization for Research and Treatment of Cancer (EORTC). Int J Radiat Oncol Biol Phys 1995; 30: 1341-6. 